\title{
Extinction and permanence of the predator-prey system with general functional response and impulsive control ${ }^{\sqrt{k}}$
}

\author{
Juan $\mathrm{Liu}^{\mathrm{a}}, \mathrm{Jie} \mathrm{Hu}^{\mathrm{b}}$, Peter Yuen ${ }^{\mathrm{c}, *}$ \\ ${ }^{a}$ College of Arts and Sciences, Shanxi Agricultural University, Taigu 030801, PR China \\ ${ }^{b}$ School of software, Shanxi Agricultural University, Taigu 030801, PR China \\ ${ }^{c}$ Centre for Electronics Warfare, Information and Cyber (CEWIC) \\ Cranfield University, Shrivenham, Swindon SN6 8LA U.K.
}

\begin{abstract}
Traditional approach for modelling the evolution of populations in the predatorprey ecosystem has commonly been undertaken using specific impulsive response function, and this kind of modelling is applicable only for a specific ecosystem under certain environmental situations only. This paper attempts to fill the gap by modelling the predator-prey ecosystem using a 'generalized' impulsive response function for the first time. Different from previous research, the present work develops the modelling for an integrated pest management (IPM) especially when the stocking of predator (natural enemy) and the harvesting of prey (pest) occur impulsively and at different instances of time. The paper firstly establishes the sufficient conditions for the local and the global stabilities of prey eradication periodic solution by applying the Floquet theorem of the Impulsive different equation and small amplitude perturbation under a 'generalized' impulsive response function. Subsequently the sufficient condition for the permanence of the system is given through the comparison techniques. The corollaries of the theorems that are established by using the 'general impulsive response function' under the locally asymptotically stable condition are found to be in excellent agreement with those reported previously. Theoretical results that are obtained in this work is then validated by using a typical impulsive
\end{abstract}

\footnotetext{
${ }^{\text {th }}$ Fully documented templates are available in the elsarticle package on CTAN.

* Corresponding author

Email address: p.yuen@cranfield.ac.uk (Peter Yuen)
} 
response function (Holling type-II) as an example, and the outcome is shown to be consistent with the previously reported results. Finally, the implication of the developed theories for practical pest management is illustrated through numerical simulation. It is shown that the elimination of either the preys or the pest can be effectively deployed by making use of the theoretical model established in this work. The developed model is capable to predict the population evolutions of the predator-prey ecosystem to accommodate requirements such as: the combinations of the biological control, chemical control, any functional response function, the moderate impulsive period, the harvest rate for the prey and predator parameter and the incremental stocking of the predator parameter. Keywords: Impulsive, General functional response, Globally asymptotically stable, Permanence

2010 MSC: 00-01, 99-00

\section{Introduction}

Study of dynamic systems has been an important research subject due to its diverse applications across vast multi-disciplinary science and engineering such as for the modeling of biological systems, communication, control system$\mathrm{s}$, networked systems, manufacturing and mechanical systems etc[1, 2]. The dynamic system is said to be hybrid when it exhibits continuous and discrete dynamics[3, 4], and it is termed as a switched system when it consists of a family of subsystems in which the dynamics is switched among the subsystems according to a logical rule[2, $5-10]$. The interactions of prey and predator, such as the study of the populations in the crops and pests system, is a simplified special case of the dynamic systems which has been investigated extensively in the ecological, agricultural and environmental research within the past few decades[11-15]. Particularly, the research on the pest control has been a popular topic as it imposes significant impacts on the social and economic stability of the region in question[16-18]. There are two commonly used approaches for pests control: chemically through pesticide spray and biological control through 
the introduction of natural enemies into the local environment. In some cases the integrated pest management (IPM) which utilizes both chemical and the environmentally friendly biological control[16-19] is preferred, particularly when results are desired to be achieved rapidly. However, to maintain the long term existences of prey or the eliminations of the pest, it requires the delicate balance between when the cycles of pesticides should be applied and the exact timing for introducing natural enemies. This is needed to be evaluated through careful modeling or experiments due to the negative effect of pesticide to the natural enemies of the pest. Due to the difficulty to implement experiments effectively in the ecological systems, mathematical modeling has been an alternative promising way to solve practical problems[1-38]. Considering the discontinuity of the spraying pesticides and natural enemies, the description of the short-term rapid changes of their populations is commonly modeled through impulsive differential equations (IDE) [8, 14-35]. Most previous work modelled the simultaneously spraying pesticides and natural enemies impulsively at the same fixed moments $[8,22,27]$. It is not reasonable due to the effects of pesticides on natural enemies. Thus the modelling of the predator-prey system using non-simultaneous timings of impulsive control strategies is more realistic and relevant for practical applications.

The predator-prey relationship between pests and natural enemies is quite well-known. Typical models involve a characteristic predators rate of feeding on the prey, which is known as the functional response, to model the change of predation rate as a function of the prey density through a set of differential equations. Depending on the nature of ecosystems concerned, many different kinds of functional responses such as the Holling type-I $\left(\phi_{1}\right)$, the Holling type-II $\left(\phi_{2}\right)$, the Holling type-III $\left(\phi_{3}\right)[15,19-21]$ have been applied for various applications. The Holling type $\phi_{1}, \phi_{2}$ and $\phi_{3}$ are commonly in the form of:

$$
\phi_{1}(x)=\left\{\begin{array}{ll}
\frac{b}{a} x & 0<x<a \\
b & x>a
\end{array} \quad, \quad \phi_{2}(x)=\frac{r x}{a+b x}, \quad \phi_{3}(x)=\frac{r x^{2}}{a+b x^{2}} .\right.
$$


The Ivlev-type functional response $\phi_{4}$ is in the form of $[22,30]$ :

$$
\phi_{4}(x)=r\left(1-e^{-\alpha x}\right) .
$$

where a,b,r, $\alpha$ are constants and $x(t)$ is prey density. Note that the above functional responses are modelled for scenarios when the predator-prey interaction depends on prey densities only. In the real world both the prey and predator densities affect the predator-prey interactions, so more complex functional response such as the Beddington-DeAngelis functional response $\phi_{5}$ has been proposed [8]:

$$
\phi_{5}(x, y)=\frac{m x}{a+b x+c y} .
$$

where $y(t)$ represents the density of the predator, and alternative form of the ratio-dependent functional response $\phi_{6}$ has also been suggested [15, 23, 25]:

$$
\phi_{6}\left(\frac{x}{y}\right)=\frac{\frac{c x}{y}}{m+\frac{x}{y}}=\frac{c x}{m y+x} .
$$

where a,b,m,c are constants. Due to the complicated natural environment which compounds by various degree of human interferences, a plethora of functional responses has been proposed for modelling the dynamical relationships between the predator and the prey, for example[26-29, 31]:

the Watt-type functional response $\phi_{7}=\exp \frac{-c x}{y^{m}}$;

the Hassell-Varley functional response $\phi_{8}=\frac{c x(t)}{m y^{\gamma}+x}$;

the Square-Root functional responses $\phi_{9}=\frac{\beta \sqrt{x(t)}}{1+\alpha \sqrt{x(t)}}$;

the Monod-Haldance functional response $\phi_{10}=\frac{m x(t)}{a+x^{2}(t)}$ etc.

Instead of modelling the predator-prey system using a specific type of functional response like that performed by previous research, this paper attempts for the first time to model the predator-prey system by using a general functional response. This paper gives a generalized local and global stability of prey eradication periodic solution under sufficient conditions, and the solution is then illustrated by using a specific functional response as an example.

Previous work employed the Holling II functional response [24], the MonodHaldance functional response [29, 31], the Ivlev-type functional response [30], 
the ratio-dependent functional response [15, 23], the Beddington-DeAngelis functional response [8] and the Hassell-Varley functional response etc [27], for modelling the predator-prey system using these impulsive control strategies. Although these previous studies had provided the local asymptotic stability of prey-eradication periodic solution and the permanence of system under sufficient conditions, they could be applied only for specific scenarios and therefore their impacts to the real world is limited.

Thus it is necessary to generalize previous findings such as that reported in $[24,29,30]$ by using a general functional response for modelling the predatorprey system, especially when the predator is stocked and the prey is harvested at different instances of time. It is believed that this work may represent a first study of its kind for modelling the predator-prey system by using the general function $g(x, y)$ as the system in (1) below:

$$
\left\{\begin{array}{l}
\frac{\mathrm{d} x(t)}{\mathrm{d} t}=x(t)\left(r-\frac{r}{K} x(t)\right)-g(x, y) x(t) y(t) \\
\frac{\mathrm{d} y(t)}{\mathrm{d} t}=y(t)(-D+k g(x, y) x(t)) \\
\left.\begin{array}{rl}
\Delta x(t) & =-p_{1} x(t) \\
\Delta y(t) & =-p_{2} y(t)
\end{array}\right\} t=(n+l-1) T \\
\left.\begin{array}{rl}
\Delta x(t) & =0 \\
\Delta y(t) & =\mu
\end{array}\right\} t=n T
\end{array}\right.
$$

where $x(t), y(t)$ denote the densities of the prey (pest) and predator (natural enemy) at time $t$, respectively. $T$ is the period of the impulsive harvesting and stocking. $r>0$ is the intrinsic growth rate of the prey, $K>0$ is the carrying capacities of the prey, $D>0$ is the death rate of the predator. The $k>0$ denotes the rate of converting consumed preys into the growth of predator, and the $x(t) g(x, y)$ is the general functional response of the prey which represents the rate of predation by the predator per-capita. The $g(x, y)$ is assumed to be monotonous decreasing with respect to $x$ and $y$ respectively, which satisfied the Lemma 2.3 in Section 2, and $y(t) g(x, y)$ is the monotonous increasing density of $y$ which represents the main functional responses of the ecosystem. Also, 
$\Delta x(t)=x\left(t^{+}\right)-x(t) ; \Delta y(t)=y\left(t^{+}\right)-y(t) ; x\left(t^{+}\right)=\lim _{t \rightarrow t^{+}} x(t) ; y\left(t^{+}\right)=$ $\lim _{t \rightarrow t^{+}} y(t)$. And $0 \leq p_{1}, p_{2}<1$ is the harvest rate of the prey and the predator at time $(n+l-1) T$ respectively, where $l \in(0,1), n \in N . \mu>0$ is the incremental stocking of the predator at time $n T$.

This paper is organized as follows: Section 1 (this section) outlines the problem statement and the objectives of this paper. The preliminaries including definitions and lemmas are given in Section 2. In Section 3, the stability of the prey-eradication periodic solution, including the local asymptotic stability and global asymptotically stability of the predator-prey system modelled by using a 'general' functional response is given. Section 4 investigates the boundedness of solutions and the sufficient conditions that are required for the permanence of the system (1). To illustrate the established theoretical results, numerical simulations of the systems in section 3 have been performed by using a specific response function as an example and the results are presented in Section 5. Section 6 summarizes the methodology of the present work to model the prey and predator system using a generalized impulsive response function, and the suggestions to apply the results of this work for practical pest management are subsequently given.

\section{Definitions and Lemmas}

In this section, the lemmas and definitions which are needed in this work are given here:

Definition 2.1. [39] The system (1) is said to be permanent provided that there exists positive constants $m$ and $M$, such that each positive solution $(x(t), y(t))$ of the system (1) satisfies $m \leq x(t) \leq M, m \leq y(t) \leq M$ for all large enough $t$.

Definition 2.2. [39] The prey or the predator goes extinct provided that there exists a solution $(x(t), y(t))$ such that $\lim _{t \rightarrow \infty} x(t)=0$ or $\lim _{t \rightarrow \infty} y(t)=0$.

Lemma 2.3. [39] The solution of system (1) is a piecewise continuous function. Denote by $f=\left(f_{1}, f_{2}\right)$ the right-hand sides of the first, second equations of 
system (1). The smoothness properties of $f$ guarantee the uniqueness and global existence of the solutions of the system (1).

Considering the following subsystem of system (1) when $x=0$ :

$$
\begin{cases}\frac{d y(t)}{d t}=-D y(t), & t \neq(n+l-1) T, t \neq n T \\ y\left(t^{+}\right)=\left(1-p_{2}\right) y(t), & t=(n+l-1) T \\ y\left(t^{+}\right)=y(t)+\mu, & t=n T \\ y_{0}=y\left(0^{+}\right) & \end{cases}
$$

By employing the stroboscopic mapping and the fixed-point theorem, the following positive periodic solution of the system in (2) can be obtained:

$$
\tilde{y}(t)= \begin{cases}\frac{\mu \exp \{-D[t-(n-1) T]\}}{1-\left(1-p_{2}\right) \exp (-D T)}, & (n-1) T<t \leq(n+l-1) T \\ \frac{\mu\left(1-p_{2}\right) \exp \{-D[t-(n-1) T]\}}{1-\left(1-p_{2}\right) \exp (-D T)}, & (n+l-1) T<t \leq n T .\end{cases}
$$

and $\tilde{y}\left(0^{+}\right)=\tilde{y}\left(n T^{+}\right)=\frac{\mu}{1-\left(1-p_{2}\right) \exp (-D T)}, \tilde{y}\left(l T^{+}\right)=\tilde{y}\left((n+l-1) T^{+}\right)=$ $\frac{\mu\left(1-p_{2}\right) \exp (-D l T)}{1-\left(1-p_{2}\right) \exp (-D T)}$. The solution of the system in (2) with initial value $y_{0} \geq 0$ can then be obtained:

$$
y(t)=\left\{\begin{array}{l}
\left(1-p_{2}\right)^{n-1}\left(y\left(0^{+}\right)-\frac{\mu}{1-\left(1-p_{2}\right) \exp (-D T)}\right) \exp (-D t)+\tilde{y}(t), \\
(n-1) T<t \leq(n+l-1) T, \\
\left(1-p_{2}\right)^{n}\left(y\left(0^{+}\right)-\frac{\mu}{1-\left(1-p_{2}\right) \exp (-D T)}\right) \exp (-D t)+\tilde{y}(t), \\
(n+l-1) T<t \leq n T .
\end{array}\right.
$$

Lemma 2.4. [39] Let $X(t)=(x(t), y(t))$ be any solution of system (1), then $X(t) \geq 0$ holds for all $t \geq 0$ if $X\left(0^{+}\right) \geq 0$ and $X(t)>0$ holds for all $t \geq 0$ if $X\left(0^{+}\right)>0$.

Lemma 2.5. [39] For every positive solution of system (2) with initial value $y_{0} \geq 0$, then we have $|y(t)-\tilde{y}(t)| \rightarrow 0$ as $t \rightarrow \infty$.

In the following, we define $g\left(x_{0}, y_{0}\right)=\lim _{\substack{x \rightarrow x_{0} \\ y \rightarrow y_{0}}} g(x, y)$, where $x(t) g(x, y)$ is general functional response, for example in the case of the Ivlev-type functional 
response then

$$
g(x, y)=\frac{r\left(1-e^{-\alpha x}\right)}{x}
$$

which is not well defined at $(0,0)$ and so the

$$
g\left(x_{0}, y_{0}\right)=\lim _{\substack{x \rightarrow 0 \\ y \rightarrow 0}} \frac{r\left(1-e^{-\alpha x}\right)}{x}=r \alpha
$$

is defined instead.

Therefore, the positive prey-eradication periodic solution $(0, \tilde{y}(t))$ can be obtained.

\section{The stability of prey-eradication periodic solution}

Theorem 3.1. Let $X(t)=(x(t), y(t))$ the solution of the system in (1), then the prey-eradication periodic solution $(0, \tilde{y}(t))$ is locally asymptotically stable if

$$
\ln \left(1-p_{1}\right)+r T-\int_{0}^{T} g(0, \tilde{y}(s)) \tilde{y}(s) d s<0
$$

Proof. By applying the small amplitude perturbations of the solution and the use of the Floquet theorem, the local stability of the periodic solution $(0, \tilde{y}(t))$ can be obtained.

Firstly, define $u(t)=x(t), w(t)=y(t)-\tilde{y}(t)$, where $u(t), w(t)$ are small perturbations. Let the system be expanded in the Taylor series and after neglecting the higher order terms and only consider the linear part:

$$
\left\{\begin{array}{l}
\left.\begin{array}{l}
\frac{d u(t)}{d t}=u(t)(r-g(0, \tilde{y}) \tilde{y}) \\
\frac{d w(t)}{d t}=-D w(t)+k g(0, \tilde{y}) \tilde{y} u(t)
\end{array}\right\} t \neq(n+l-1) T, t \neq n T \\
u\left((n+l-1) T^{+}\right)=\left(1-p_{1}\right) u((n+l-1) T) \\
w\left((n+l-1) T^{+}\right)=\left(1-p_{2}\right) w((n+l-1) T)
\end{array}\right\} t=(n+l-1) T
$$


Let $\phi(t)$ be the fundamental matrix of (5), then $\phi(t)$ satisfies:

$$
\frac{d \phi(t)}{d t}=\left(\begin{array}{cc}
r-g(0, \tilde{y}) \tilde{y} & 0 \\
k g(0, \tilde{y}) \tilde{y} & -D
\end{array}\right) \phi(t) .
$$

The solution of the above gives:

$$
\phi(t)=\left(\begin{array}{cc}
\exp \left(\int_{0}^{t}(r-g(0, \tilde{y}) \tilde{y}) d s\right) & 0 \\
* & \exp (-D t)
\end{array}\right) .
$$

It is not necessary to evaluate the exact form of $(*)$ as it is not required in the analysis that follows. The linearization of the resetting impulsive conditions of the system in (5) gives:

$$
\begin{gathered}
\left(\begin{array}{c}
u\left((n+l-1) T^{+}\right) \\
w\left((n+l-1) T^{+}\right)
\end{array}\right)=\left(\begin{array}{cc}
1-p_{1} & 0 \\
0 & 1-p_{2}
\end{array}\right)\left(\begin{array}{c}
u((n+l-1) T) \\
w((n+l-1) T)
\end{array}\right), \\
\left(\begin{array}{c}
u\left(\mathrm{n} T^{+}\right) \\
w\left(\mathrm{n} T^{+}\right)
\end{array}\right)=\left(\begin{array}{ll}
1 & 0 \\
0 & 1
\end{array}\right)\left(\begin{array}{l}
u(\mathrm{n} T) \\
w(\mathrm{n} T)
\end{array}\right) .
\end{gathered}
$$

Thus the monodromy matric of the system in (5) is in the form of:

$$
M=\left(\begin{array}{cc}
1-p_{1} & 0 \\
0 & 1-p_{2}
\end{array}\right)\left(\begin{array}{ll}
1 & 0 \\
0 & 1
\end{array}\right) \phi(T) .
$$

Let $\lambda_{1}, \lambda_{2}$ be eigenvalues of matrix $M$, then we have:

$$
\begin{aligned}
& \lambda_{1}=\left(1-p_{1}\right) \exp \left(\int_{0}^{T}(r-g(0, \tilde{y}) \tilde{y}) d s\right), \\
& \lambda_{2}=\left(1-p_{2}\right) \exp (-D T)<1 .
\end{aligned}
$$

By using the Floquent theory of impulsive different equation, the prey-eradication periodic solution $(0, \tilde{y}(t))$ is local asymptotically stable if $\left|\lambda_{1}\right|<1$, that is

$$
\ln \left(1-p_{1}\right)+r T-\int_{0}^{T} g(0, \tilde{y}(s)) \tilde{y}(s) d s<0 .
$$

This completes the proof.

Theorem 3.2. Let $X(t)=(x(t), y(t))$ be the solution of the system in (1), then the prey-eradication periodic solution $(0, \tilde{y}(t))$ is globally asymptotically stable if

$$
\ln \left(1-p_{1}\right)+r T-\int_{0}^{T} g(K, \tilde{y}(s)) \tilde{y}(s) d s<0 .
$$


Proof. Noticing that:

$$
\begin{cases}\frac{\mathrm{d} x(t)}{\mathrm{d} t} \leq x(t)\left(r-\frac{r}{K} x(t)\right), & t \neq(n+l-1) T, t \neq n T, \\ x\left(t^{+}\right)=\left(1-p_{1}\right) x(t) \leq x(t), & t=(n+l-1) T, t=n T .\end{cases}
$$

Considering the following impulsive different equation:

$$
\left\{\begin{array}{l}
\frac{\mathrm{d} u(t)}{\mathrm{d} t}=u(t)\left(r-\frac{r}{K} u(t)\right) \\
u\left(0^{+}\right)=x_{0}
\end{array}\right.
$$

From the comparison theorem, we have $x(t) \leq u(t)$. Then

$$
u(t)=\frac{K x_{0}}{\left(K-x_{0}\right) e^{-r_{1} t}+x_{0}} \rightarrow K(t \rightarrow \infty) .
$$

Therefore $x(t) \leq K+\varepsilon_{1}$ holds for any $\varepsilon_{1}>0$ and all large enough $t$. For simplification and without loss of generality, we may assume that $x(t) \leq K+\varepsilon_{1}$ holds for all $t>0$.

Select an $\varepsilon>0$, such that

$$
\eta=\left(1-p_{1}\right) \exp \left(\int_{0}^{T} r-g\left(K+\varepsilon_{1}, \tilde{y}(s)-\varepsilon\right)(\tilde{y}(s)-\varepsilon) d s\right) \in(0,1) .
$$

From the second equation of the system (1) we get that $\frac{\mathrm{dy}(t)}{\mathrm{d} t} \geq-D y(t)$, thus $y(t) \geq \tilde{y}(t)$. Using the Lemma 2.5, we have $y(t) \rightarrow \tilde{y}(t)(t \rightarrow \infty)$, then for sufficiently large t:

$$
y(t) \geq \tilde{y}(t)>\tilde{y}(t)-\varepsilon
$$

Similarly by assuming (8) holds for all $t>0$. It follows from the system (1) that:

$$
\frac{\mathrm{d} x}{\mathrm{~d} t} \leq x\left(r-g\left(K+\varepsilon_{1}, \tilde{y}(s)-\varepsilon\right)(\tilde{y}(s)-\varepsilon)\right),
$$

which leads to:

$$
\begin{aligned}
x((n+l) T) & \leq x\left((n+l-1) T^{+}\right) A \\
& =x((n+l-1) T)\left(1-p_{1}\right) A \\
& =x((n+l-1) T) \cdot \eta .
\end{aligned}
$$


where $A=\exp \left(\int_{(n+l-1) T}^{(n+l) T} r-g\left(K+\varepsilon_{1}, \tilde{y}(s)-\varepsilon\right)(\tilde{y}(s)-\varepsilon) d s\right)$. Thus

$$
x((n+l) T) \leq x(l T) \eta^{n} \rightarrow 0(n \rightarrow \infty) .
$$

Since

$$
0 \leq x(t) \leq x((n+l-1) T)\left(1-p_{1}\right) e^{r T} .
$$

And it holds for $t \in[(n+l-1) T,(n+l) T]$, therefore $x(t) \rightarrow 0$ when $t \rightarrow \infty$.

In the following, we prove that $y(t) \rightarrow \tilde{y}(t)$ provided that $x(t) \rightarrow 0$ as $t \rightarrow \infty$. For any sufficiently small $\varepsilon_{2}>0$, there exists a $T>0$ such that $0<x(t)<\varepsilon_{2}$ and $t>T$, then we have:

$$
-D y(t) \leq \frac{\mathrm{d} y(t)}{\mathrm{d} t} \leq y(t)\left(-D+k \varepsilon_{2} g(0, \tilde{y}(t)-\varepsilon)\right)
$$

By the comparison techniques, we get:

$$
\tilde{y}_{1}(t) \leq y(t) \leq \tilde{y}_{2}(t)
$$

where $\tilde{y}_{1}(t)$ and $\tilde{y}_{2}(t)$ are the positive periodic solutions of the following impulsive different equation:

$$
\begin{cases}\frac{d y(t)}{d t}=-D y(t), & t \neq(n+l-1) T, t \neq n T \\ y\left(t^{+}\right)=\left(1-p_{2}\right) y(t), & t=(n+l-1) T \\ y\left(t^{+}\right)=y(t)+\mu, & t=n T \\ y_{0}=y\left(0^{+}\right) & \end{cases}
$$

and

$$
\begin{cases}\frac{d y(t)}{d t}=\left(-D+k \varepsilon_{2} g(0, \tilde{y}(t)-\varepsilon)\right) y(t), & t \neq(n+l-1) T, t \neq n T \\ y\left(t^{+}\right)=\left(1-p_{2}\right) y(t), & t=(n+l-1) T \\ y\left(t^{+}\right)=y(t)+\mu, & t=n T \\ y_{0}=y\left(0^{+}\right) & \end{cases}
$$

From the Lemma 2.5, we obtain:

$$
\tilde{y}_{1}(t) \rightarrow \tilde{y}(t), \tilde{y}_{2}(t) \rightarrow \tilde{y}(t)
$$


for any large enough $t$. It follows from (9) that $y(t) \rightarrow \tilde{y}(t)$ when $t \rightarrow \infty$. This completes the proof.

As according to theorem 3.1 if we let $g(x, y)$ respectively to be:

$$
\begin{aligned}
g(x, y)=\frac{a}{b+c x(t)}, g(x, y) & =\frac{m}{a+x^{2}(t)}, g(x, y)=\frac{h\left(1-e^{-c x(t)}\right)}{x(t)}, \\
g(x, y) & =\frac{c}{m y(t)+x(t)} .
\end{aligned}
$$

then the following corollary can be obtained:

Corollary 3.3. The prey-eradication periodic solution $(0, \tilde{y}(t))$ is locally asymptotically stable if

$$
\begin{aligned}
& \ln \left(1-p_{1}\right)+r T-\frac{a A}{b}<0 . \\
& \ln \left(1-p_{1}\right)+r T-\frac{m A}{a}<0 . \\
& \ln \left(1-p_{1}\right)+r T-h c A<0 . \\
& \ln \left(1-p_{1}\right)+r T-\frac{c T}{m}<0 .
\end{aligned}
$$

where $A \triangleq \int_{0}^{T} \tilde{y}(s) d s=\frac{\mu\left[1-p_{2} \exp (-D l T)-\left(1-p_{2}\right) \exp (-D T)\right]}{d\left[1-\left(1-p_{2}\right) \exp (-D T)\right]}$.

Remark 3.4. The inequalities (10), (11), (12) and (13) of Corollary 3.3 are exactly identical to the theorem 3.1 in [24], the theorem 3.2 in [29], the theorem 3.1 in [30], and the theorem 3.1 in [23]. This may show that the theorems 3.1 and 3.2 derived in the above sections are indeed the generalized results for the general impulsive response $g(x, y)$.

\section{Boundedness and permanence}

Theorem 4.1. Let $X(t)=(x(t), y(t))$ be any solution of the system in (1), then there exists a constant $M>0$, such that $x(t) \leq M, y(t) \leq M$ for all sufficiently large $t$. 
Proof. Define a function $W(t, x)=k x(t)+y(t)$, then:

$$
D^{+} W(t, x)=k \frac{\mathrm{d} x(t)}{\mathrm{d} t}+\frac{\mathrm{d} y(t)}{\mathrm{d} t}=k x(t)\left(r-\frac{r}{K} x(t)\right)-D y(t) .
$$

which is the upper right derivative of $W(t, x)$ along a solution of the system in (1). For $0<\lambda \leq D$, we have

$$
\begin{aligned}
& D^{+} W(t, x)+\lambda W(t, x)=k x(t)\left(\lambda+r-\frac{r}{K} x(t)\right)+(\lambda-D) y(t) \\
& \leq k(\lambda+r) x-\frac{r k x^{2}}{K}=-\frac{k r}{K}\left(x^{2}-\frac{(\lambda+r) K x}{r}\right) \\
& =-\frac{k r}{K}\left[\left(x-\frac{(\lambda+r) K}{2 r}\right)^{2}-\frac{(\lambda+r)^{2} K^{2}}{4 r^{2}}\right] \leq \frac{(\lambda+r)^{2} K k}{4 r} .
\end{aligned}
$$

Thus there exists a positive constant number $M_{1}=\frac{(\lambda+r)^{2} K k}{4 r}$, such that

$$
D^{+} W(t, x)+\lambda W(t, x) \leq M_{1}
$$

and when $t=n T$, we get $W\left(n T^{+}\right) \leq W(n T)+\mu$, and when $t=(n+l-1) T$, we also have $W\left((n+l-1) T^{+}\right) \leq W((n+l-1) T)$. Then according to Lemma 2.4 in [39], we have:

$$
\begin{aligned}
W(t) & \leq W(0) e^{-\lambda t}+\frac{M_{1}}{\lambda}\left(1-e^{-\lambda t}\right)+\frac{\mu e^{-\lambda(t-T)}}{1-e^{-\lambda T}}+\frac{\mu e^{\lambda T}}{e^{\lambda T}-1} \\
& \rightarrow \frac{M_{1}}{\lambda}+\frac{\mu e^{\lambda T}}{e^{\lambda T}-1}(t \rightarrow \infty) .
\end{aligned}
$$

Hence $W(t, x)$ is ultimately bounded by a constant and $M=\frac{M_{1}}{\lambda}+\frac{\mu e^{\lambda T}}{e^{\lambda T}-1}$. The proof is completed.

Theorem 4.2. The system in (1) is permanent provided that

$$
\ln \left(1-p_{1}\right)+r T-\int_{0}^{T} g(0, \tilde{y}(s)) \tilde{y}(s) d s>0
$$

Proof. As according to the Theorem 4.1, it has been proved that there exists a constant number $M>0$, such that $x(t) \leq M, y(t) \leq M$ for all large enough $t$.

Let

$$
m=\frac{\mu\left(1-p_{2}\right) \exp (-D T)}{1-\left(1-p_{2}\right) \exp (-D T)}-\varepsilon>0 .
$$

Thus it is easy to deduce from Lemma 2.5 that $y(t)>m$ for sufficiently large $t$.

Next, it is needed to show that there exist $m_{0}>0$ such that $x(t)>m_{0}$ for sufficiently large $t$. The proof can be shown in two steps: 


\section{A. Step 1}

Firstly, by choosing a sufficiently small constant $\varepsilon_{1}>0$ and $0<m_{1}<\frac{D}{k g(0, m)}$ such that

$$
\sigma=\ln \left(1-p_{1}\right)+\left(r-\frac{r}{K} m_{1}\right) T-\int_{0}^{T} g\left(0, \tilde{z}(t)+\varepsilon_{1}\right)\left(\tilde{z}(t)+\varepsilon_{1}\right) d t>0 .
$$

It is known that $m_{1}$ is not unique. For a given $t_{1}>0$ a claim that $x\left(t_{1}\right)>m_{1}$ may or may not be valid, if it is not then for any $t>0$ such that $x(t) \leq m_{1}$. It follows from the system in (1) that

$$
\frac{\mathrm{d} y(t)}{\mathrm{d} t} \leq y(t)\left(-D+k m_{1} g(0, m)\right) .
$$

By using the comparison theorem, we can get $y(t) \leq z(t), z(t) \rightarrow \tilde{z}(t)$ as $t \rightarrow \infty$, where $z(t)$ is the solution of the following impulsive differential equation:

$$
\begin{cases}\frac{d z(t)}{d t}=\left(-D+k m_{1} g(0, m)\right) z(t), & t \neq(n+l-1) T, t \neq n T \\ z\left(t^{+}\right)=\left(1-p_{2}\right) z(t), & t=(n+l-1) T \\ z\left(t^{+}\right)=z(t)+\mu, & t=n T \\ z_{0}=y\left(0^{+}\right) & \end{cases}
$$

and

$\tilde{z}(t)= \begin{cases}\frac{\mu \exp \left\{\left(-D+k m_{1} g(0, m)\right)[t-(n-1) T]\right\}}{1-\left(1-p_{2}\right) \exp \left[\left(-D+k m_{1} g(0, m)\right) T\right]}, & (n-1) T<t \leq(n+l-1) T \\ \frac{\mu\left(1-p_{2}\right) \exp \left\{\left(-D+k m_{1} g(0, m)\right)[t-(n-1) T]\right\}}{1-\left(1-p_{2}\right) \exp \left[\left(-D+k m_{1} g(0, m)\right) T\right]}, & (n+l-1) T<t \leq n T .\end{cases}$

Therefore there exists $T_{1}>0$ such that $y(t) \leq z(t) \leq \tilde{z}(t)+\varepsilon_{1}$, and

$$
\frac{\mathrm{d} x(t)}{\mathrm{d} t} \geq x(t)\left(r-\frac{r}{K} m_{1}-g\left(0, \tilde{z}(t)+\varepsilon_{1}\right)\left(\tilde{z}(t)+\varepsilon_{1}\right)\right) .
$$

Integrate (15) in the limits $[(n+l-1) T,(n+l) T]$ gives:

$$
\begin{aligned}
x((n+l) T) & \geq x\left((n+l-1) T^{+}\right) A \\
& =x((n+l-1) T)\left(1-p_{1}\right) A \\
& =x((n+l-1) T) \exp (\sigma) .
\end{aligned}
$$

where $A=\exp \left(\int_{(n+l-1) T}^{(n+l) T} r-\frac{r}{K} m_{1}-g\left(0, \tilde{z}(t)+\varepsilon_{1}\right)\left(\tilde{z}(t)+\varepsilon_{1}\right) d s\right)$. Thus

$$
x((n+l) T) \geq x(l T) \exp (n \sigma) \rightarrow \infty,
$$


as $n \rightarrow \infty$, which is in contradiction to the boundedness of $x(t)$ given in the theorem 3 .

\section{B. Step 2}

Next, it is needed to prove that $x(t)>m_{1}$ which holds for all $t>t_{1}$. If it is true, then we are done. Otherwise $x(t) \leq m_{1}$ for some $t>t_{1}$.

Let

$$
t^{*}=\inf _{t \geq t_{1}}\left\{x(t)<m_{1}\right\},
$$

we have $x\left(t_{1}\right) \geq m_{1}$ for $t \in\left[t_{1}, t^{*}\right)$ and $t^{*} \in\left[n_{1} T,\left(n_{1}+1\right) T\right], n_{1} \in N$. Since $x(t)$ is continuous, we can get $x\left(t^{*}\right)=m_{1}$. Choose $n_{2}, n_{3} \in N$ such that:

$$
\begin{gathered}
n_{2} T>\frac{1}{\left(-D+k m_{1} g(0, m)\right)} \ln \frac{\varepsilon_{1}}{M+\mu}, \\
\quad \exp \left(\delta\left(n_{2}+1\right) T\right) \exp \left(n_{3} \sigma\right)>1,
\end{gathered}
$$

in which $\delta \triangleq r-\frac{r}{K} m_{1}-g(0, M) M<0$.

By setting $T^{\prime}=\left(n_{2}+n_{3}\right) T$, it can be claimed that there exists $t_{2} \in$ $\left[\left(n_{1}+1\right) T,\left(n_{1}+1\right) T+T^{\prime}\right]$ such that $x\left(t_{2}\right) \geq m_{1}$. Otherwise $x(t)<m_{1}$ and from (14) with $z\left(\left(n_{1}+1\right) T^{+}\right)=y\left(\left(n_{1}+1\right) T^{+}\right)$, we obtain:

$$
z(t)=\left\{\begin{array}{l}
\left(1-p_{2}\right)^{n-1}\left(z\left(\left(n_{1}+1\right) T^{+}\right)-\frac{\mu}{1-\left(1-p_{2}\right) \exp \left[\left(-D+k m_{1} g(0, m)\right) T\right]}\right) * \\
\exp \left[\left(-D+k m_{1} g(0, m)\right)\left(t-\left(n_{1}+1\right) T\right)\right]+\tilde{z}(t) \\
\left(1-p_{2}\right)^{n}\left(z\left(\left(n_{1}+1\right) T^{+}\right)-\frac{\mu}{1-\left(1-p_{2}\right) \exp \left[\left(-D+k m_{1} g(0, m)\right) T\right]}\right) * \\
\exp \left[\left(-D+k m_{1} g(0, m)\right)\left(t-\left(n_{1}+1\right) T\right)\right]+\tilde{z}(t) .
\end{array}\right.
$$

Then

$$
|z(t)-\tilde{z}(t)|<(M+\mu) \exp \left[\left(-D+k m_{1} g(0, m)\right)\left(t-\left(n_{1}+1\right) T\right)\right]<\varepsilon_{1},
$$

and

$$
y(t) \leq z(t) \leq \tilde{z}(t)+\varepsilon_{1},
$$

which holds for

$$
\left(n_{1}+n_{2}+1\right) T \leq t \leq\left(n_{1}+1\right) T+T^{\prime}
$$


And this implies that (15) will hold for $\left(n_{1}+n_{2}+1\right) T \leq t \leq\left(n_{1}+1\right) T+T^{\prime}$, then

$$
x\left(\left(n_{1}+n_{2}+n_{3}+1\right) T\right) \geq x\left(\left(n_{1}+n_{2}+1\right) T\right) \exp \left(n_{3} \sigma\right) .
$$

There are two possible cases for $t \in\left(t^{*},\left(n_{1}+1\right) T\right]$.

Case 1:

If $x(t) \leq m_{1}$ for $t \in\left(t^{*},\left(n_{1}+1\right) T\right]$, then when $t \in\left(t^{*},\left(n_{1}+n_{2}+1\right) T\right]$, we have $x(t) \leq m_{1}$. From the system in (1), we have :

$$
\frac{\mathrm{d} x(t)}{\mathrm{d} t} \geq x(t)\left(r-\frac{r}{K} m_{1}-g(0, M) M\right) \triangleq \delta x(t) .
$$

By integrating (17) in the limit $\left(t^{*},\left(n_{1}+n_{2}+1\right) T\right]$ which yields:

$$
x\left(\left(n_{1}+n_{2}+1\right) T\right) \geq m_{1} \exp \left(\delta\left(n_{2}+1\right) T\right)
$$

Then by (16) and (18), we have:

$$
x\left(\left(n_{1}+n_{2}+n_{3}+1\right) T\right) \geq m_{1} \exp \left(\delta\left(n_{2}+1\right) T\right) \exp \left(n_{3} \sigma\right)>m_{1},
$$

which contradicts the priori condition of $x(t) \leq m_{1}$ for all $t \in\left(t^{*},\left(n_{1}+n_{2}+1\right) T\right]$.

By setting $\bar{t}=\inf _{t \geq t^{*}}\left\{x(t) \geq m_{1}\right\}$, then $x(\bar{t})=m_{1}$. Since (17) holds for $t \in\left[t^{*}, \bar{t}\right)$ and to integrate in $\left[t^{*}, \bar{t}\right)$ we get

$$
x(t) \geq x\left(t^{*}\right) \exp \left(\delta\left(t-t^{*}\right)\right) \geq m_{1} \exp \left(\delta\left(n_{2}+n_{3}+1\right) T\right) \triangleq m_{0} .
$$

Since $x(\bar{t}) \geq m_{1}$ and the same argument can be continued for $t>\bar{t}$, thus $x(t) \geq m_{0}$ for all $t>t_{1}$.

Case 2:

There is $t^{\prime} \in\left(t^{*},\left(n_{1}+1\right) T\right]$ such that $x\left(t^{\prime}\right)>m_{1}$. Put $\hat{t}=\inf _{t \geq t^{*}}\left\{x(t) \geq m_{1}\right\}$, then $x(t)<m_{1}$ when $t \in\left[t^{*}, \hat{t}\right)$ and $x(\hat{t})=m_{1}$. Note that (17) holds for $t \in\left[t^{*}, \hat{t}\right)$ and therefore we have:

$$
x(t) \geq x\left(t^{*}\right) \exp \left(\delta\left(t-t^{*}\right)\right) \geq m_{1} \exp (\delta T) \geq m_{0} .
$$

This process can be continued as $x(t) \geq m_{0}$, therefore we have $x(t) \geq m_{0}$ for all $t \geq t_{1}$. 
Thus it can be concluded in both cases $x(t) \geq m_{0}$ and it holds for all $t \geq t_{1}$. This completes the proof.

In Theorem 4.2, if we let $\mathrm{g}(\mathrm{x}, \mathrm{y})$ to be in the following forms respectively:

$$
\begin{aligned}
g(x, y)=\frac{a}{b+c x(t)}, g(x, y) & =\frac{m}{a+x^{2}(t)}, g(x, y)=\frac{h\left(1-e^{-c x(t)}\right)}{x(t)}, \\
g(x, y) & =\frac{c}{m y(t)+x(t)} .
\end{aligned}
$$

Then the following corollary can be obtained:

Corollary 4.3. The system in (1) is permanent provided that

$$
\begin{aligned}
& \ln \left(1-p_{1}\right)+r T-\frac{a A}{b}>0 . \\
& \ln \left(1-p_{1}\right)+r T-\frac{m A}{a}>0 . \\
& \ln \left(1-p_{1}\right)+r T-h c A>0 . \\
& \ln \left(1-p_{1}\right)+r T-\frac{c T}{m}>0 .
\end{aligned}
$$

Remark 4.4. The inequality (19), (20), (21) and (22) of the corollary 4.3 are identical to the theorem 3.2 in [24], the theorem 3.3 in [29], the theorem 3.2 in [30], and also the theorem 3.3 of literature [23] respectively. This again shows that the above results in theorem 4.2 are the generalized solutions for modelling the predator-prey system using the general $g(x, y)$ implusive response function.

\section{Numerical simulations}

This section is devoted to the validation of the theoretical results obtained in the above sections with respected to the findings reported in $[24,29,30]$ through numerical simulations. 
Firstly, let $f(T)=\ln \left(1-p_{1}\right)+r T-\int_{0}^{T} g(0, \tilde{y}(s)) \tilde{y}(s) d s$, then $f(0)=$ $\ln \left(1-p_{1}\right), f(T) \rightarrow \infty$ as $T \rightarrow \infty$. As $f^{\prime \prime}(T)>0, f(T)=0$ has an unique positive root $T_{\max }$, which is the critical value of $T$.

It is known from Theorem 3.1 and Theorem 4.2 that when $T<T_{\max }$, the system in (1) has the asymptotically stable periodic solution for the prey eradication, otherwise the system in (1) is permanent. It can be seen that the system is either permanent or locally asymptotically stable, which conforms to the actual predator-prey relationship in the biological systems.

Secondly, if $g(x, y)=\frac{a}{b+c x}$, where $g(x, y)$ is the Holling type-II functional response, it can be concluded that the prey-eradication periodic solution is locally and globally asymptotically stable if

$$
\ln \left(1-p_{1}\right)+r T-\frac{a A}{b}<0
$$

and

$$
\ln \left(1-p_{1}\right)+r T-\frac{a A}{b+c K}<0
$$

as according to the Theorem 3.1 and Theorem 3.2 respectively.

The condition of the locally stability is seen dependent on the given initial value, but there is no limitation on the global stability. Therefore the condition of the global stability is stricter than that of the locally stability, which is also in consistent with the conclusions drawn by the Theorem 3.1 and Theorem 3.2 above. It has also been indicated that the system is in permanent condition provided that $\ln \left(1-p_{1}\right)+r T-\frac{a A}{b}>0$ as shown in the Theorem 4.2.

Thirdly, if the following parametrization is used: $r=3, K=1.5, d=$ $0.4, p_{1}=0.5, p_{2}=0.1, u=8, l=0.5, k=0.5, a=0.4, b=0.4, c=0.6$; then we can obtain $T_{\max }=7.3$ and it satisfies the conditions of Theorem 3.1 when $T=6$ with initial value $x(0)=1, y(0)=0$ and the prey eradication periodic solution is locally asymptotically stable.

Fig.1 illustrates the existence of the prey eradication and it exhibits the prey density is reduced to zero rapidly (in Fig.1(a)) while the predator population oscillates ceaselessly (Fig.1(b)). Fig.1(c) is the phase portrait of $x(t)$ and $y(t)$ 

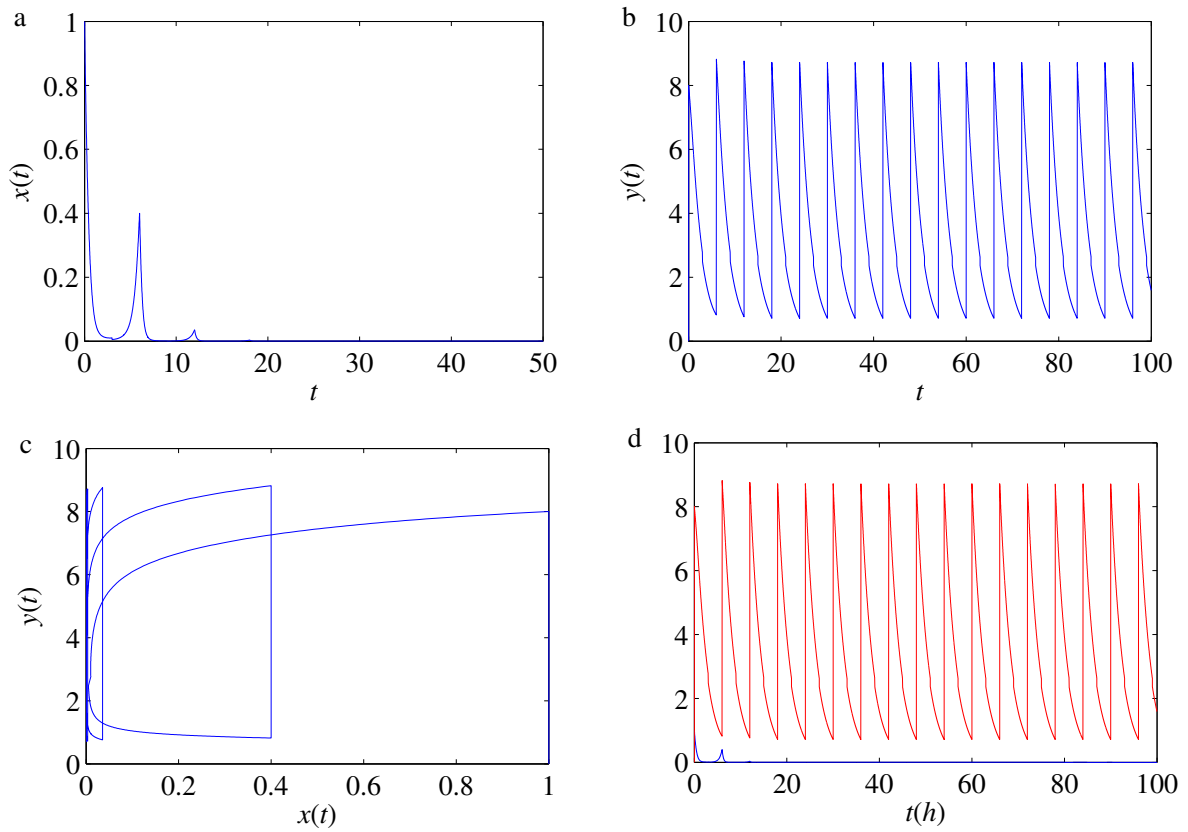

Fig. 1: Illustrates the dynamical behavior of the system (1) with $T=6$ : (a) The time sequence diagram of the prey population $x(t)$. (b) The time sequence diagram of the predator population $y(t)$. (c) The phase diagram of $x(t)$ and $y(t)$ which tends to stable gradually, and (d) the time sequence diagram of the $x(t)$ and $y(t)$ by overlaying (a) onto (b), where the red line is $y(t)$, the blue line is $x(t)$.

which tends to approaching a stable state gradually. Fig.1(d) overlays the plots in (a) and (b) for better visualization of results. Similarly, the system in (1) is permanent, i.e. when the predator and the prey can coexist as it is shown in Fig 2(a) and (b) when these parameters fulfill the conditions as stated in the Theorem 4.2. As discussed above it shows that the dynamic behavior of the system in (1) is more complex particularly when the impulsive period is changed. This implies that a moderate impulsive period can be adopted to suit for different control objectives or strategies.

Finally, consider the cases of the system in (1) when it is subjected to the single biological and single chemical control situations. In the single biological control, i.e. when the harvest rate for the prey and predator $p_{1}=p_{2}=0$ and 

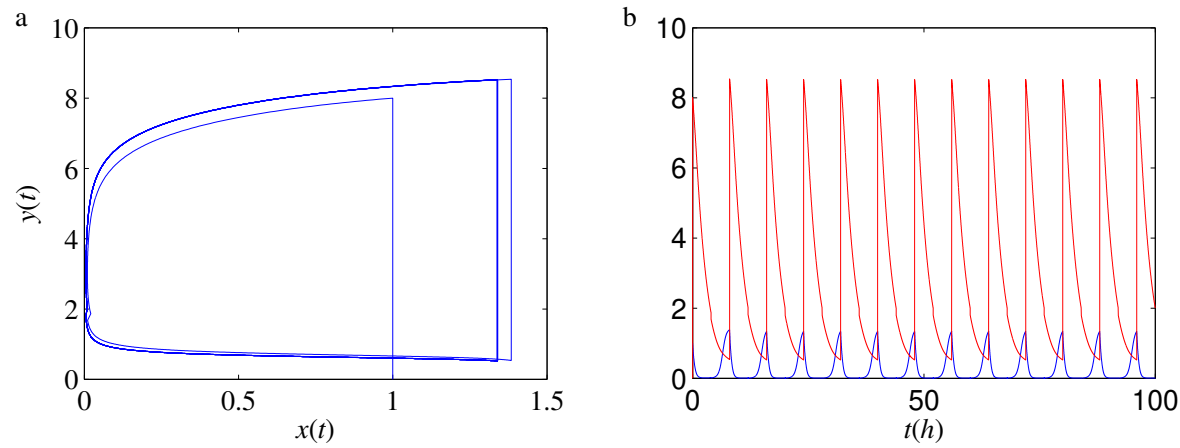

Fig. 2: Graphically illustrates the dynamical behavior of the system in (1) with $T=8$. (a) Shows the phase diagram of $x(t)$ and $y(t)$ and (b) The time sequence diagram of $x(t)$ and $y(t)$, where the red line is $y(t)$, the blue line is $x(t)$.
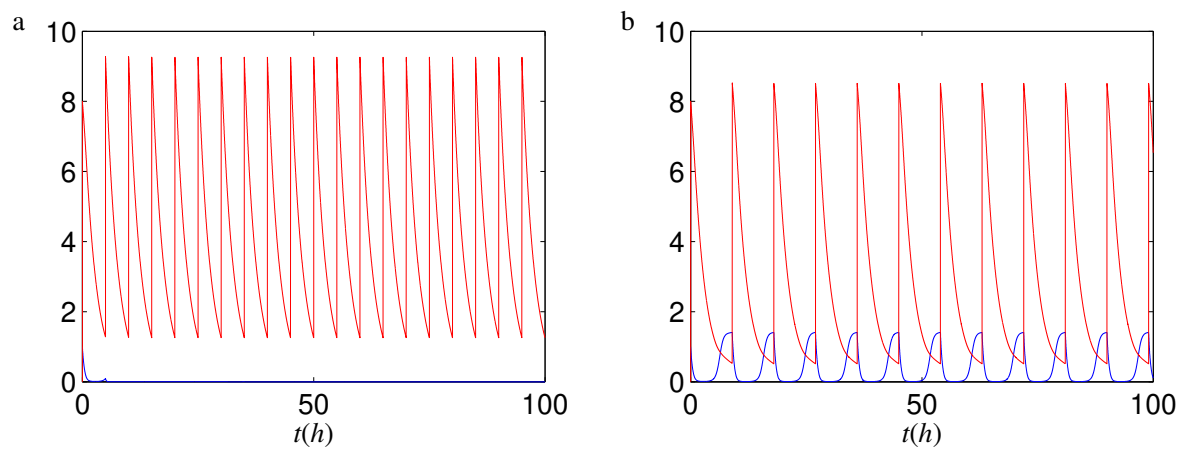

Fig. 3: Dynamical behavior of the system in (1) under the single biological control, i.e. $p_{1}=p_{2}=0$. (a)The prey-eradication periodic solution of the single biological control when $T=5$. (b)The permanence of the single biological control when $T=9$, where the red line is $y(t)$, the blue line is $x(t)$. 

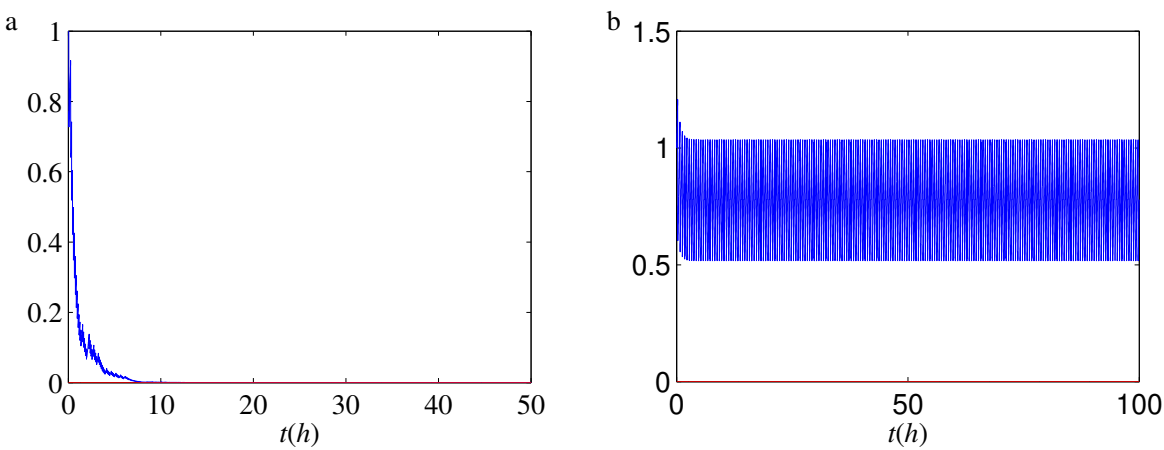

Fig. 4: Dynamical behavior of system (1) with the single chemical control, i.e. when $\mu=0$. (a)The prey-eradication periodic solution of the single biological control when $T=0.1$. (b)The permanence of the single biological control when $T=0.5$, where the red line is $y(t)$, the blue line is $x(t)$.

$T_{\max }=6.66$ is obtained as according to the inequality (4). When the system (1) is subjected to the single chemical control, i.e. when $\mu=0$ then $T_{\max }=0.23$ is obtained.

Fig.3 and Fig.4 plot the asymptotic stability and permanence of the system in (1) under the single biological and chemical controls respectively by using two different values of $\mathrm{T}$ and the same parametrization as that employed in Fig.1. By comparing the $T_{\max }$ of the single biological control (i.e. $T_{\max }=6.66$ ) and single chemical controls (i.e. $T_{\max }=0.23$ ) with respected to that of the integrated pest management (i.e. $T_{\max }=7.3$ ) as illustrated in Fig.1, it is obvious that the integrated pest management which exhibits higher $T_{\max }$ indicative of the more effectiveness for controlling the pest than that using the single control method.

\section{Conclusions}

This paper attempts for the first time to model the predator-prey system by using a generalized impulsive response function. The sufficient condition of locally asymptotic stability of prey-eradication periodic solution and the permanence of this system have been established according to the Theorem 3.1 and Theorem 4.2 derived in this work. These theorems are in fact the extension of 
existing achievements reported in recent papers by Liu et al and Baek et al in $[24,29,30]$. The theoretical results obtained in this work is then validated by using a typical impulsive response function (Holling type-II) as an example, and the outcome is shown consistent with the previously reported results [24]. Furthermore, the present work has investigated more complex dynamic behaviors including the global asymptotic stability in Theorem 3.2, which had not been studied in many previous work $[24,29,30]$.

The end result of the present work can be applied widely to any impulsive predator-prey system for any specific functional responses. The theories that have been established here can be served as an effective and practical pest management guide for real world applications. Dependent on the different situations of the practical farmland, the pest can be eliminated and environmental pollution can be reduced by treating the system using a moderate impulsive period, a desire harvest rate for the prey and predator through the parameter $p i(i=1,2)$ and a user selected incremental stocking of the predator parameter $\mu$.

\section{Acknowledgements}

The authors would like to thank the financial supports by the Shanxi Agricultural University of Science and Technology Innovation Fund Projects(2017019, 2017005).

\section{References}

[1] M. Zhang, L. Gao, Input-to-state stability for impulsive switched nonlinear systems with unstable subsystems, Transactions of the Institute of Measurement and Control 40(7)(2018)2167-2177.

[2] H. Zhu, P. Li, X. Li, H. Akca, Input-to-state stability for impulsive switched systems with incommensurate impulsive switching signals, Communications in Nonlinear Science and Numerical Simulation 80(2020)104969.

[3] J. Yuan, X. Zhang, X. Zhu, E. Feng, H. Yin, Z. Xiu, Pathway identification 
using parallel optimization for a nonlinear hybrid system in batch culture, Nonlinear Analysis: Hybrid Systems 15(2015)112-131.

[4] L. Gao, M. Zhang, X. Yao, Stochastic input-to-state stability for impulsive switched stochastic nonlinear systems with multiple jumps, International Journal of Systems Science 50(2019)1860-1871.

[5] J. Yuan, C. Wu, J. Ye, J. Xie, Robust identification of nonlinear statedependent impulsive switched system with switching duration constraints, Nonlinear Analysis: Hybrid Systems 36(2020)100879.

[6] J. Yuan, J. Xie, C. Liu, K. Teo, M. Huang, H. Fan, E. Feng, Z. Xiu, Robust optimization for a nonlinear switched time-delay system with noisy output measurements using hybrid optimization algorithm, Journal of the Franklin Institute 356(2019)9730-9762.

[7] C. Liu, Z. Gong, H. Wing, J. Lee, K. Teo, Robust bi-objective optimal control of 1, 3-propanediol microbial batch production process, Journal of Process Control 78(2019)170-182.

[8] Z. Yao, S. Xie, N. Yu, Dynamics of cooperative predator-prey system with impulsive effects and Beddington-DeAngelis functional response, Journal of the Egyptian Mathematical Society 21(2013)213-223.

[9] J. Yuan, Y. Zhang, J. Ye, J. Xie, K. Teo, X. Zhu, E. Feng, H, Yin, Z. Xi$\mathrm{u}$, Robust parameter identification using parallel global optimization for a batch nonlinear enzyme-catalytic time-delayed process presenting metabolic discontinuities, Applied Mathematical Modelling 00(2017)1-24.

[10] J. Yuan, J. Xie, M. Huang, H. Fan, E. Feng, Z, Xiu, Robust optimal control problem with multiple characteristic time points in the objective for a batch nonlinear time-varying process using parallel global optimization, Optimization and Engineering https://link.springer.com/article/10.1007 \%2Fs11081-019-09472-z. 
[11] Z. Wang, Y. Xie, J. Lu, Y. Li, Stability and bifurcation of a delayed generalized fractional-order prey-predator model with interspecific competition, Applied Mathematics and Computation 347(2019)360-369.

[12] X. Wang, Z. Wang, H. Shen, Dynamical analysis of a discrete-time SIS epidemic model on complex networks, Applied Mathematics Letters 94(2019) 292-299.

[13] A. Pervez, P. Singh, H. Bozdoğan, Ecological perspective of the diversity of functional responses, European Journal of Environmental Sciences 8(2018)97-101.

[14] Z. Wang, Y. Shao, X. Fang, The dynamic behaviors of one-predator twoprey system with mutual interference and impulsive control, Mathematics and Computers in Simulation 132(2017)68-65.

[15] W. Li, J. Ji, L. Huang, Global dynamic behavior of a predator-prey model under ratio-dependent state impulsive control, Applied Mathematical Modelling 77(2020)1842-1859.

[16] J. Hu, J. Liu, Q. Zhao, Dynamic analysis of pest-epidemic model with impulsive control, Mathematics in practice and theory 49(2)(2019)304-310.

[17] J. Li, X. Zhu, X. Lin, J. Li, Impact of cannibalism on dynamics of a structured predator-prey system, Applied Mathematical Modelling 78(2020)119.

[18] J. Hu, J. Liu, A novel pulsing rodent control method equipped with ratio-dependent reaction system, Journal of Shanxi Agricultural University(Nature Science Edition) 38(9)(2018)37-42.

[19] C.V. Kumar, K. S. Reddy, M. Srinivas, Dynamics of prey predator with Holling interactions and stochastic influences, Alexandria Engineering Journal 57(2018)1079-1086. 
[20] A. Izquierdo, M. Len, M. Mayado, A generalized Holling type II model for the interaction between dextral-sinistral snails and Pareas snakes, Applied Mathematical Modelling 73(2019)459-472.

[21] S. Li, W. Liu, A delayed Holling type III functional response predatorprey system with impulsive perturbation on the prey, Advance in different equations 42(2016)42-62.

[22] X. Wang, J. Wei, Dynamics in a diffusive predator-prey system with strong Allee effect and Ivlev-type functional response, Journal of Mathematical Analysis and Applications 422(2015)1447-1462.

[23] J. Liu, J. Hu, Q. Zhao, Seasonally perturbed ratio-dependent predator-prey system with impulsive control, Journal of Biomathematics 33(2)(2018)160170.

[24] B. Liu, Z. Teng, L. Chen, Analysis of a predator-prey model with Holling II functional response concerning impulsive control strategy, Journal of Computational and Applied Mathematics 193(2006)347-362.

[25] Z. Liu, L. Zhang, P. Bi, J. Pang, B. Li, C.Fang, On the dynamics of one-prey-n-predator impulsive reaction-diffusion predator-prey system with ratio-dependent functional response, Journal of Biological Dynamics 12(2018)551-576.

[26] X. Lin, Y.Jiang, X.Wang, Existence of periodic solutions in predator-prey with Watt-type functional response and impulsive effects, Nonlinear Analysis: Theory, Methods and Applications 73(6)(2010)1684-1697.

[27] H. Kim, H. Baek, The dynamical complexity of a predator-prey system with Hassell-Varley functional response and impulsive effect, Mathematics and Computers in Simulation 94(2013)1-14.

[28] X. Wang, C. Huang, Permanence of a stage-structured predator-prey system with impulsive stocking prey and harvesting predator, Applied Mathematics and Computation 235(2014)32-42. 
[29] Z.Liu, R.Tan, Impulsive harvesting and stocking in a Monod-Haldane functional response predator-prey system, Chaos Solitons Fractals 34(2007)454464.

[30] H. Baek, S. Kim, P. Kim, Permanence and stability of an Ivlev-type predator-prey system with impulsive control strategies, Applied Mathematical Modelling 50(2009)1385-1393.

[31] C. Ling, S. Tang, Seasonally perturbed the Monod-Haldance type predatorprey model with impulsive, Journal of Biomathematics 28(2013)499-504.

[32] Z. Shi, H. Cheng, Y. Li, Y. Li, A Cydia Pomonella Integrated Management Predator-Prey Model With Smith Growth and Linear Feedback Control, IEEE Access 7(2019)126066-126076.

[33] A. Pervez, P. Singh, H. Bozdoğan, Ecological perspective of the diversity of functional responses, European Journal of Environmental Sciences 8(2018)97-101.

[34] J. Liu, Q. Zhao, C. Chen, Stability of two prey and one predator systems with impulsive effects, Jiangsu agricultural science 46(18)(2018)94-97.

[35] J. Liu, J. Hu, Q. Zhao, Dynamic analysis of the predator-prey system in a polluted environment with impulsive, Mathematics in practice and theory 49(22)(2019)294-298.

[36] Y. Xie, J. Liu, Z. Wang, Stability analysis of a fractional-order diffused prey-predator model with prey refuges, Physica A: Statistical Mechanics and its Applications 526(2019)120773.

[37] G. Wang, Y. Liu, J. Lu, Z. Wang, Stability analysis of totally positive switched linear systems with average dwell time switching, Nonlinear Analysis: Hybrid Systems 36(2020)100877.

[38] J. Yuan, X. Zhang, C. Liu, L. Chang, J. Xie, E. Feng, H. Yin, Z. Xi$\mathrm{u}$, Robust optimization for nonlinear time-delay dynamical system of dha 
regulon with cost sensitivity constraint in batch culture, Communication in Nonlinear Science and Numerical Simulation 38(2016)140-171.

[39] V. Lakshmikantham, D. Bainov, S. Simeonov, Theory of Impulsive Differential Equations, World Scientific Publisher: Singapore, 1989. 